\title{
Effects of metformin on FOXM1 expression and on the biological behavior of acute leukemia cell lines
}

\author{
BING ZHANG ${ }^{1,2}$, LONG-LONG LIU ${ }^{3}$, XIA MAO $^{1}$ and DONG-HUA ZHANG ${ }^{1}$ \\ ${ }^{1}$ Department of Hematology, Tongji Hospital, Tongji Medical College, Huazhong University of Science and Technology, \\ Wuhan, Hubei 430030; ${ }^{2}$ Department of Hematology, Jiangsu Province Hospital of TCM, Affiliated Hospital of \\ Nanjing University of TCM, Nanjing, Jiangsu 210000; ${ }^{3}$ Department of Hematology, Wuhan General \\ Hospital of Guangzhou Military Command, Wuhan, Hubei 430070, P.R. China
}

Received November 30, 2013; Accepted June 17, 2014

DOI: $10.3892 / \mathrm{mmr} .2014 .2629$

\begin{abstract}
Forkhead box M1 (FOXM1) is a typical proliferation-associated transcription factor, which is overexpressed in many types of human cancer. We investigated the expression level of FOXM1 in patients with untreated acute leukemia (AL) and explored the correlation between expression levels and AL type. The relationship between the expression of the genes FOXM1 and mammalian target of rapamycin (mTOR) was determined after treatment of ML-2 cells with thiostrepton. The apoptosis, proliferation and cell-cycle progression of ML-2 lines were examined after treatment with metformin. We found that FOXM1 is expressed in the majority of $\mathrm{AL}$ patients and that its expression level was associated with the AL type. Thiostrepton is a specific inhibitor of FOXM1, and by inhibiting the FOXM1 expression via thiostrepton, we observed downregulatiion of mTOR; a significant correlation between FOXM1 and mTOR levels was observed. Thus, metformin may be involved in the downregulation of FOXM1. In addition, our study demonstrated that metformin promotes the apoptosis of ML-2 cells, induces cell-cycle arrest at the G0/G1 and G2/M phases, and inhibits proliferation. The potential role of FOXM1 in tumorigenesis renders it an attractive target for anticancer therapy, and metformin may represent a new agent for the treatment of leukemia.
\end{abstract}

\section{Introduction}

Forkhead box M1 (FOXM1) is a typical proliferation-associated transcription factor that stimulates cell proliferation and exhibits a proliferation-specific expression pattern. FOXM1 is highly expressed in numerous solid tumors, such as lung,

Correspondence to: Professor Dong-Hua Zhang, Department of Hematology, Tongji Hospital, Tongji Medical College, Huazhong University of Science and Technology, 1095 Jiefang Road, Wuhan, Hubei 430030, P.R. China

E-mail: zdh62@aliyun.com

Key words: FOXM1, mTOR, metformin, acute myeloid leukemia, thiostrepton breast, prostate and pancreatic cancer (1-3). FOXM1 is intimately involved in tumorigenesis, contributing to oncogenic transformation and participating in tumor initiation, growth and progression. Nakamura et al first reported that FOXM1 is overexpressed in acute myeloid leukemia (AML) cells, especially in cells with high aldehyde dehydrogenase activity (4).

An important aspect of the present study is the detection of the expression level of FOXM1 in untreated acute leukemia (AL) patients, and the analysis of the relationship between FOXM1 expression and the expression or the mutational status of other genes, in order to determine its prognostic value in AL. Another major aspect of our study is the investigation of the effects of metformin. Epidemiological evidence suggests that patients with diabetes have a high incidence of cancer. However, the cancer risk is lower in diabetic patients who were treated with metformin. A reasonable explanation for this finding is that metformin may have antitumor effects (5). Previous studies have demonstrated that metformin can activate the AMP-activated protein (AMPK) kinase pathway and inhibit the mammalian target of rapamycin (mTOR) to decrease the expression of Bcl-2 and c-Myc proteins $(6,7)$. Metformin also was also shown to accelerate tumor cell apoptosis by inhibiting the phosphorylation of the signal transducer and activator of transcription (STAT3) (8).

In the current study, the effects of metformin on the biological behavior of ML-2 cells were analyzed. We aimed to confirm the antitumor function of metformin in AL patients.

\section{Materials and methods}

Sample preparation. Bone marrow samples were obtained from 134 untreated AL patients. Of these, 107 were diagnosed with AML, and 27 with acute lymphoblastic leukemia (ALL). Diagnosis was performed at the Tonji Hospital of Tonji Medical College (Wuhan, China), based on criteria proposed by the French-American-British (FAB) Group. Written informed consent was obtained from the subjects. The mononuclear cells were separated from the bone marrow and stored at $-80^{\circ} \mathrm{C}$. The study was approved by the Ethical Committee of Tonji Hospital, Tonji Medical College, Huazhong University of Science and Technology (Wuhan, China) and was conducted according to the principles of the Declaration of Helsinki. The 
AML cell lines Kassumi, NB-4, THP-1, ML-2, K562, and the ALL lines Jurkat, Raji and HUT-78 were purchased from the Shanghai Cell Bank (Chinese Academy of Sciences, Shanghai, China). The cell lines were maintained in RPMI-1640 medium, supplemented with Hyclone ${ }^{\mathrm{TM}} 10 \%$ fetal bovine serum (FBS; Thermo Fisher Scientific, Waltham, MA, USA) at $37^{\circ} \mathrm{C}$ with $5 \% \mathrm{CO}_{2}$.

Reverse transcription (RT)-semiquantitative and quantitative $(q)$ polymerase chain reaction $(P C R)$. The cells were collected after treatment for $16 \mathrm{~h}$ with 5,10 and $20 \mu \mathrm{mol}$ of thiostrepton (Enzo Biochem, New York, NY, USA). RNA was extracted from these cells using the RNeasy Mini kit (Qiagen, Hilden, Germany), and an aliquot was reverse transcribed into cDNA using the RevertAid ${ }^{\mathrm{TM}}$ First Strand cDNA Synthesis kit (Fermentas, Vilnius, Lithuania). The RT-PCR primers were as follows: FOXM1 forward (F), 5'-CGAAAGATGAGTTCTGAT GG-3', and reverse (R), 5'-GAAAGGTTGTGGCGGATG-3'; FMS-like tyrosine kinase 3 internal tandem duplication (FLT3/ITD) F, 5'-TGTCGAGCAGTACTCTAAACA-3', and R, 5'-ATCCTAGTACCTTCCCAAACTC-3'; GAPDH F, 5'-CCA CCCATGGCAAATTCCATGGCA-3' and R, 5'-TCTAGACGG CAGGTCAGG-3'. The qRT-PCR primers were as follows: FOXM1 F, 5'-TGCCCAGCAGTCTCTTACCT-3', and R, 5'-CTACCCACCTTCTGGCAGTC-3'; GAPDH F, 5'-GCA CCGTCAAGGCTGAGAAC-3', and R, 5'-TGGTGAAGACGC CAGTGGA-3'; mTOR F, 5'-CGCTGTCATCCCTTTATCG-3', and R, 5'-ATGCTCAAACACCTCCACC-3'. All primers were purchased from Invitrogen Life Technologies (Shanghai, China). The RT-PCR reactions for FOXM1 and GAPDH were performed in $20-\mu l$ volumes. The cycling program consisted of a 5 -min pre-denaturation at $94^{\circ} \mathrm{C}$, followed by 32 cycles of denaturation at $94^{\circ} \mathrm{C}$ for $50 \mathrm{sec}$, annealing at $58^{\circ} \mathrm{C}$ for $1 \mathrm{~min}$, and extension at $72^{\circ} \mathrm{C}$ for $30 \mathrm{sec}$. qRT-PCR of FOXM1, mTOR and GAPDH was performed using the SYBR-Green dye Realtime PCR Master mix kit; TOYOBO, Osaka, Japan) in 10- $\mu 1$ reaction volumes. The cycling program comprised $1 \mathrm{~min}$ of pre-denaturation at $95^{\circ} \mathrm{C}$, followed by 40 cycles of denaturation at $95^{\circ} \mathrm{C}$ for $15 \mathrm{sec}$, annealing at $60^{\circ} \mathrm{C}$ for $15 \mathrm{sec}$ and extension at $72^{\circ} \mathrm{C}$ for $15 \mathrm{sec}$. The fluorescence emitted by SYBR-Green was measured and the data analyzed using a StepOne ${ }^{\mathrm{TM}}$ Real-Time PCR System (Applied Biosystems Life Technologies, Foster City, CA, USA).

Western blot analysis. The cells were collected at 3 days after treatment for $72 \mathrm{~h}$ with 2,4 and $8 \mu \mathrm{mol}$ of metformin (Sigma, St. Louis, MO, USA). The cells were lysed in RIPA buffer, containing $0.1 \%$ sodium dodecyl sulfate (SDS), $0.5 \%$ sodium deoxycholate, $1 \% \mathrm{NP}-40,150 \mathrm{mmol} / \mathrm{l} \mathrm{NaCl}, 10 \mathrm{mmol} / \mathrm{l}$ Tris- $\mathrm{HCl}$, and a cocktail of protease inhibitors by incubation for $20 \mathrm{~min}$ at $4^{\circ} \mathrm{C}$. Proteins were then quantified with a BCA Protein Assay kit (Beyotime, Shanghai, China). Total protein extracts were separated by $10 \%$ SDS-PAGE and transferred to polyvinylidene difluoride membranes. The membranes were incubated with rabbit anti-human poly-clonal primary antibody targeting FOXM1 (1:800 dilution; Proteintech Group, Chicago, IL, USA) at $4^{\circ} \mathrm{C}$ for $12 \mathrm{~h}$. After blocking in $5 \%$ fat-free milk, the membranes were incubated with horseradish peroxidase-conjugated goat anti-rabbit poly-clonal IgG secondary antibody (1: 2,000; Proteintech Group) at room temperature for $2 \mathrm{~h}$. The proteins were visualized using the ECL detection
Table I. Expression of the forkhead box M1 gene (FOXM1) in acute leukemia patients.

\begin{tabular}{lrrrc}
\hline & \multicolumn{4}{c}{ FOXM1 } \\
\cline { 2 - 5 } Type & POS & NEG & Total & Positive rate (\%) \\
\hline AML & & & & \\
M1 & 9 & 2 & 11 & 81.80 \\
M2 & 16 & 5 & 21 & 76.20 \\
M3 & 15 & 2 & 17 & 88.20 \\
M4 & 5 & 0 & 5 & 100.00 \\
M5 & 36 & 12 & 48 & 75.00 \\
M6 & 1 & 3 & 4 & 25.00 \\
M7 & 1 & 0 & 1 & 100.00 \\
Total & 83 & 24 & 107 & 77.57 \\
ALL & & & & \\
T cell & 11 & 0 & 11 & 100.00 \\
B cell & 15 & 1 & 16 & 93.75 \\
Total & 26 & 1 & 27 & 96.30 \\
Total & 109 & 25 & 134 & 81.34 \\
\hline
\end{tabular}

AML, acute myeloid leukemia; ALL, acute lymphoblastic leukemia; POS, positive; NEG, negative. $\chi^{2}=26.2, \mathrm{P}=0.004$ for all comparisons of positive rate among different AL subtypes. The AML subtypes M1-M7 refer to the FAB classification system.

system (ECL kit; Beyotime), and data were quantified with the Odyssey Infrared Imaging System (LI-COR Biosciences, Lincoln, NE, USA).

Apoptosis assay. Following treatment with metformin for 24 or $48 \mathrm{~h}$, the cells were collected, washed twice with phosphate-buffered saline (PBS), and resuspended in Annexin binding buffer (Keygen Biotech., Nanjing, China). The cells were then incubated with Annexin V-fluorescein isothiocyanate (FITC) and propidium iodide (PI) for $15 \mathrm{~min}$ in the dark using the Annexin V-FITC Apoptosis Detection kit (KeyGen Biotech.). The apoptosis rate was measured on a FACSCalibur $^{\mathrm{TM}}$ flow cytometer (BD Biosciences, San Diego, CA, USA), and analyzed with the CellQuest software (BD Biosciences, Franklin Lakes, NJ, USA).

Cell cycle analysis. Cell-cycle progression was studied by flow cytometry on PI-stained cells. The cells were collected and fixed with $70 \%$ ethanol at $-20^{\circ} \mathrm{C}$ for $12 \mathrm{~h}$ after treatment with metformin for 24 or $48 \mathrm{~h}$. Subsequently, the cells were resuspended in PBS and incubated with $10 \mathrm{mg} / \mathrm{ml}$ RNase (Fermentas, Vilnius, Lithuania) at $37^{\circ} \mathrm{C}$ for $30 \mathrm{~min}$, followed by incubation with $500 \mu \mathrm{g} / \mathrm{ml}$ PI at room temperature for $30 \mathrm{~min}$ in the dark after two PBS washes. PI-stained cells were analyzed by flow cytometry using a FACSCalibur cytometer.

Cell proliferation analysis. ML-2 cells were seeded in 24-well flat-bottom plates at a density of $2 \times 10^{5}$ cells/well, 
A

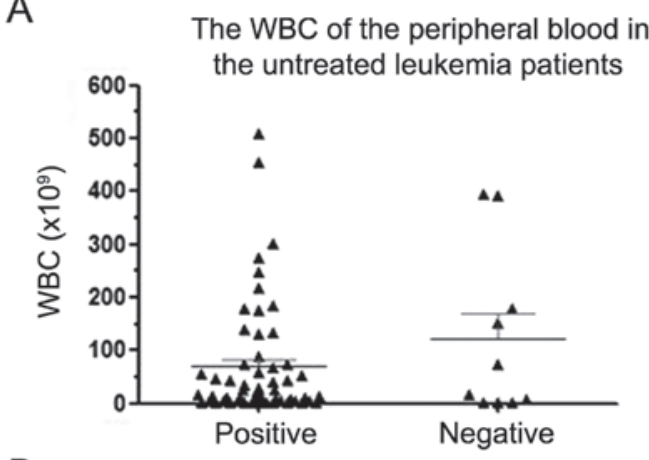

B

The blast percentage of the peripheral blood in the untreated leukemia patients

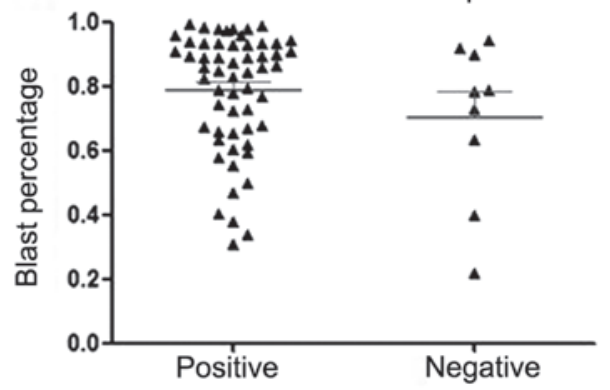

Figure 1. Expression of the forkhead box M1 (FOXM1) mRNA in mononuclear cells from untreated acute leukemia patients (AL). (A) The white blood cell (WBC) count and (B) the bone barrow myeloblast (blast)percentage in the peripheral blood of the untreated AL patients, grouped based on their FOXM1 expression status.

Table II. The relationship between the expression of the forkhead box M1 gene (FOXM1) and a mutation in the FMS-like tyrosine kinase 3 internal tandem duplication gene (FLT3/ITD) gene in acute myeloid leukemia.

\begin{tabular}{lccc}
\hline & \multicolumn{3}{c}{ FOXM1 } \\
\cline { 2 - 4 } FLT3 ITD & Positive & Negative & Total \\
\hline Positive & 27 & 0 & 27 \\
Negative & 64 & 20 & 84 \\
Total & 91 & 20 & 111 \\
\hline
\end{tabular}

$\chi^{2}=7.841, \mathrm{P}=0.030$ for the correlation between FOXM1 and FLT3/ITD

and $200 \mu 1$ of RPMI-1640 were added. The subsequent analyses were performed in the dark. The cells in each well were incubated with $1 \mu \mathrm{l}$ of $1,000 \mathrm{X}$ carboxyfluorescein succinimidyl ester (CFSE; Invitrogen Life Technologies) at $37^{\circ} \mathrm{C}$ for $10 \mathrm{~min}$. Subsequently, $400 \mu \mathrm{FBS}$ were added to stop the reaction. After resuspending in RPMI-1640 medium containing $10 \%$ FBS, the cells were treated with metformin, fixed in $4 \%$ paraformaldehyde and washed twice with PBS. The cell proliferative ability was then investigated by flow cytometry.

Statistical analysis. Quantitative data were expressed as the mean \pm standard error of the mean of at least three indepen-
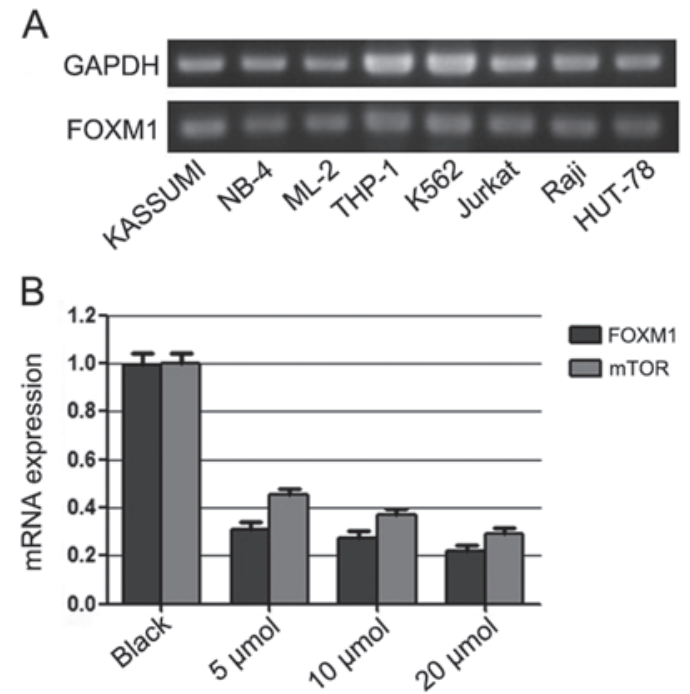

Figure 2. The effect of thiostrepton on forkhead box M1 (FOXM1) mRNA expression. (A) Gel image of reverse transcription-semiquantitative polymerase chain reaction products, showing that all the leukemia cell lines express FOXM1. (B) The expression of FOXM1 and mammalian target of rapamycin $(m T O R)$ is decreased after treatment of Jurkat cells with different concentrations of thiostrepton for $16 \mathrm{~h}$. Black, non-treated Jurkat cells.

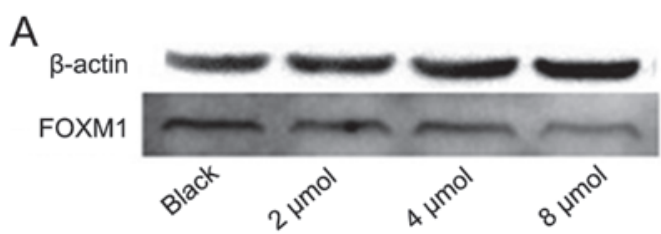

B

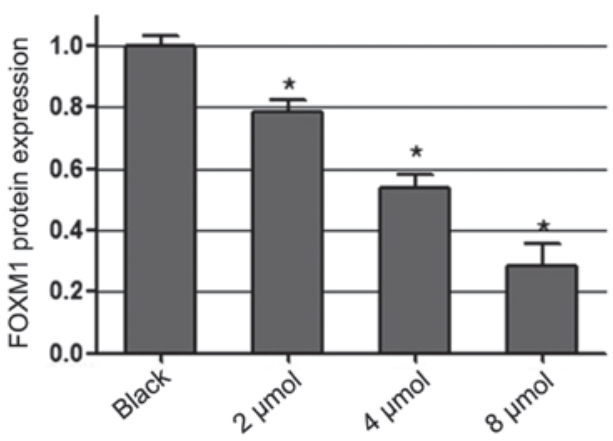

Figure 3. The effect of metformin on forkhead box M1 (FOXM1) protein expression. (A) Western blot image, with $\beta$-actin used as the loading control. (B) Metformin significantly decreases the FOXM1 protein level in a dose-dependent manner. ${ }^{*} \mathrm{P}<0.05$; black, non-treated Jurkat cells.

dent experiments. Comparisons between two groups were analyzed using Chi-square, Spearman correlation and independent samples $t$ test. A value of $\mathrm{P}<0.05$ was considered to indicate statistically significant differences. Data analysis was performed with the SPSS 15.0 software (IBM, New York, NY, USA).

\section{Results}

Expression of FOXM1 mRNA in mononuclear cells from untreated AL patients. The FOXM1 mRNA was detected by semiquantitative-PCR in $81.34 \%$ of the 134 primary $\mathrm{AL}$ patients. There was significant difference $(\mathrm{P}=0.004)$ between 
A

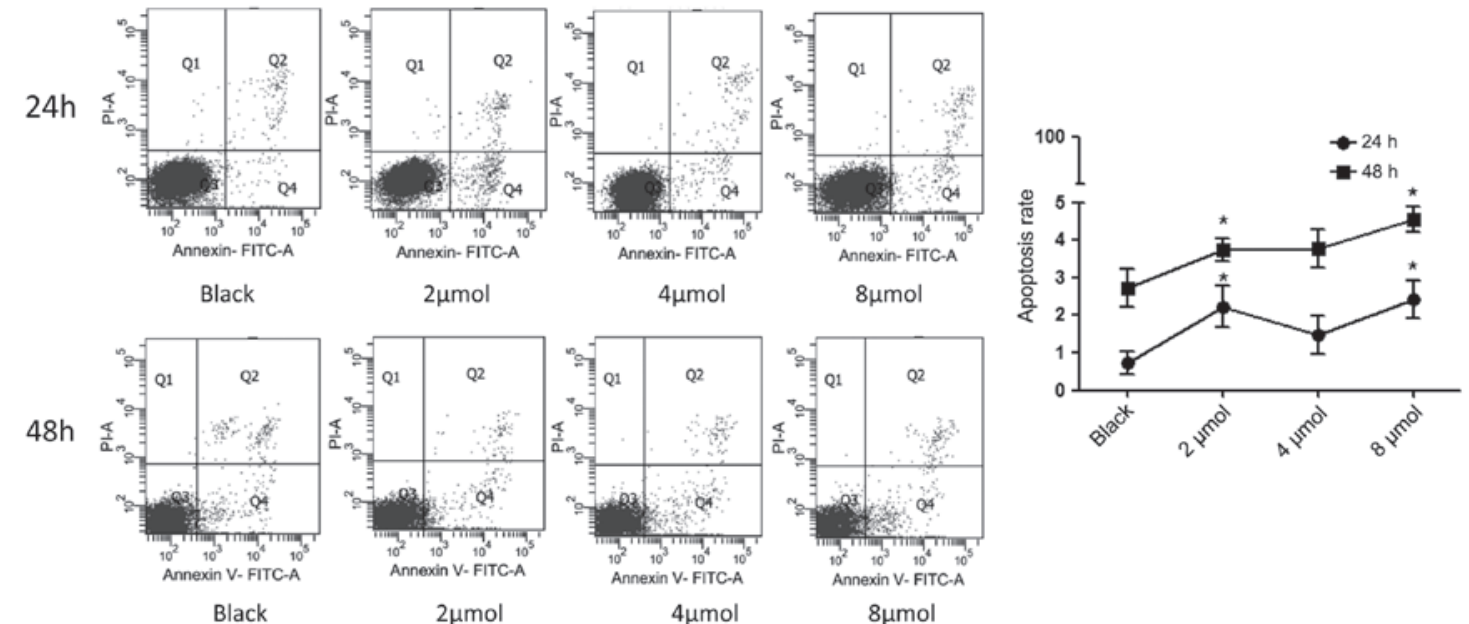

B
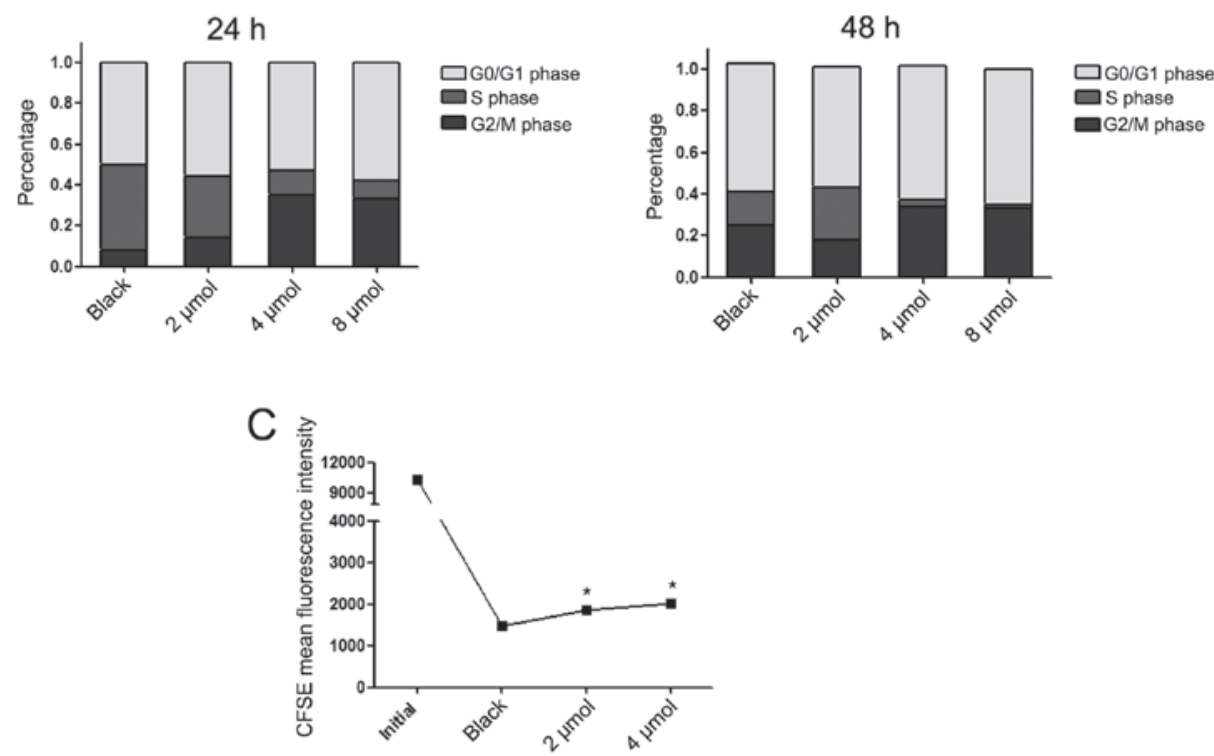

Figure 4. The biological behavior of ML-2 cells following treatment with metformin. (A) The apoptotic rate of ML-2 cells is increased after treatment for 24 and $48 \mathrm{~h}$. (B) The cell-cycle distribution of ML-2 cells following metformin treatment. FITC, fluorescein isothiocyanate; PI, propidium iodide. (C) The proliferative ability of ML-2 cells is significantly reduced, as assessed by the mean carboxyfluorescein succinimidyl ester (CFSE) fluorescence intensities. Black, non-treated cells; ${ }^{*} \mathrm{P}<0.05$.

the different subtypes with regards to the FOXMI expression status (Table I). We obtained only partial results from the analysis of patients' routine blood and bone marrow cytology tests (71/134). The white blood cell (WBC) count in the peripheral blood of the FOXM1-positive group was estimated at $69.57 \pm 110.14 \times 10^{9}$, whereas in the FOXM1-negative group, the count was $121.76 \pm 156.66 \times 10^{9}$. There was no significant difference $(\mathrm{P}=0.202)$ between the two groups (Fig. 1). Regarding the bone barrow myeloblast (also known as blast) count, the FOXM1-positive group was not significantly different from the -negative group $(78.82 \pm 18.61 \%$ vs. $67.15 \pm 24.67 \%$, $\mathrm{P}=0.120$ ). FLT3 is one of the most frequently mutated genes in AML, and is associated with poor outcome (9). In our experiments, we found that an internal tandem duplication (ITD) mutation in FLT3 correlates $(\mathrm{P}=0.030)$ to the expression of FOXM1 (Table II).

The effect of thiostrepton and metformin on FOXM1 expression. Thiostrepton is known to directly affect FOXM1 and inhibit its binding to target sites (10). In our experiments, FOXM1 was detected in all the six leukemia cell lines. Thiostrepton treatment for $16 \mathrm{~h}$ decreased the expression of both FOXM1 and $m T O R$ (Fig. 2). The Spearman correlation coefficient was $0.692(\mathrm{P}<0.05)$, indicating a positive correlation between the expression levels of ITOR and FOXM1.

The protein level of FOXM1 was analyzed after $72 \mathrm{~h}$ of incubation with metformin. In contrast to the group of non-treated cells, the FOXM1 expression levels in the 2-, 4- and $8-\mu \mathrm{mol}$ treatment groups were $0.787 \pm 0.061(\mathrm{P}<0.05$ compared to the non-treated cells), $0.537 \pm 0.081(\mathrm{P}<0.05)$ and $0.287 \pm 0.119(\mathrm{P}<0.05)$, respectively. Thus, treatment with metformin significantly decreases the FOXM1 protein level, and in a dose-dependent manner (Fig. 3).

The effect of metformin on the biological behavior of $M L-2$ cells. Following treatment with metformin for 24 and $48 \mathrm{~h}$, the rates of apoptosis of ML-2 cells in the $2-\mu \mathrm{mol}$ and $8-\mu \mathrm{mol}$ treatment groups increased. However, the $4-\mu$ mol group did 
not show the same trend (Fig. 4). These findings suggest that metformin may accelerate the apoptotic rate of ML-2 cells.

The cell-cycle distribution of ML-2 cells was determined before and after metformin treatment by Annexin-V-FITC and PI labeling, followed by flow cytometry analysis. The results indicated that metformin arrests the cell-cycle progression at the G2/M phase. However, with the exception of the 4- $\mu$ mol group, the percentage of cells at the G0/G1 and S phases decreased after $24 \mathrm{~h}$. Following $48 \mathrm{~h}$ of treatment, the cells at the $\mathrm{G} 2 / \mathrm{M}$ phase increased only in the $4-\mu \mathrm{mol}$ group $(\mathrm{P}<0.05)$.

The proliferation of ML-2 cells was examined by CFSE labeling, followed by flow cytometry analysis. The $2-\mu \mathrm{mol}$ and $4-\mu$ mol treatment groups showed lower proliferation rates after $48 \mathrm{~h}$ of treatment. These results indicate that metformin inhibits ML-2 cell proliferation.

\section{Discussion}

FOXM1 was found to be highly expressed in numerous solid tumors, such as pulmonary cancer, breast carcinoma, hepatic carcinoma, etc. Increased expression of FOXM1 indicates poor prognosis (1). In previous studies, FOXM1 was shown to accelerate the cell cycle and interfere with apoptosis $(11,12)$.

In our experiments, the FOXM1 gene was highly expressed in untreated AL patients, especially in the AML-M1, AML-M4 and T-ALL subtypes (data not shown). There was no difference between the FOXMI-positive and -negative groups with respect to the WBC of the peripheral blood and the bone marrow blast counts. We also found that FOXMI expression correlates to the FLT3 ITD mutation. It is known that AML patients with this mutation have worse outcomes than those who do not bear the mutation (13). However, an examination of the two versions of the gene revealed that the patients with high levels of FOXM1 are more likely to have an FLT3 mutation, which results in a poor prognosis, as the mutation confers an increased relapse rate and a reduction in overall survival. (13-15).

To further investigate the function of FOXM1, we used thiostrepton, which can selectively bind to FOXM1 to inhibit its transcription and translation, thereby blocking its transcription factor activity (10). To evaluate the effect of thiostrepton, we examined the expression of the genes FOXM1 and mTOR; the latter is a key regulator in the AMPK/mTOR signaling pathway. The downregulation of mTOR causes multi-site dephosphorylation of the eukaryotic translation initiation factor 4E-binding protein 1 (eIF4E-BP1), a key translational regulator, and decreases the transcription of several oncogenes, such as $c$-Myc, cyclin Dl and Bcl-xL (16). The expression of the $m T O R$ gene was decreased upon thiostrepton treatment, indicating that thiostrepton may exert antitumor activity by directly downregulating FOXM1 or by indirectly downregulating $m T O R$. The protein products of the two genes may function in the same signaling pathway.

Recently, a number of studies have demonstrated that metformin, which is widely used in the treatment of type 2 diabetes, may exert cancer chemopreventive effects in solid tumors. Metformin stimulates signaling pathways, including AMPK/mTOR, STAT3 and ERK, and restores the expression of the cell-surface major histocompatibility complex class I (16-18). Additionally, metformin may selectively target cancer stem cells
(19). However, the function of metformin in leukemia is unclear. In our study, metformin significantly decreased the protein level of FOXM1, leading to a series of biological changes.

Similar to other drugs, metformin accelerates the apoptosis of ML-2 cells and exerts antitumor activity by reducing the tumor burden. However, the exact molecular events of this process are not clear. Thus, additional in-depth studies are necessary to elucidate the cellular effects of metformin. In this study, metformin decreased the number of cells at the $S$ phase by arresting the cell cycle at the $\mathrm{G} 0 / \mathrm{G} 1$ and $\mathrm{G} 2 / \mathrm{M}$ phases. However, metformin did not affect the progression from the $\mathrm{S}$ to the $\mathrm{G} 2$ phase. It has been reported that FOXM1 affects genes that regulate the cell cycle, such as $C d c 25 A, C d c 25 B$, cyclin B, cyclin D1 and P21 (20,21), thereby arresting the cell cycle at the G0/G1 and G2 phases. mTOR has been shown to promote cell-cycle progression from the $\mathrm{G} 1$ to the $\mathrm{S}$ phase by stimulating elF-4E-dependent translation initiation (22). We hypothesize that metformin may regulate the cell cycle by decreasing the expression of $m T O R$ and FOXM1. It remains to be determined whether other genes are involved in this process. Detecting the expression of specific cyclins may allow elucidating the mechanism of cell-cycle arrest. Cell proliferation was also impaired following metformin treatment in our study. Thus, the drug may block tumor progression, and FOXM1 may be involved in this process. Overall, metformin appears to affect the apoptosis, proliferation and cell-cycle progression of AL cells, and exert an antitumor effect in vitro. The drug may function by directly or indirectly regulating the expression of FOXM1 and $m$ TOR.

Numerous clinical trials exploring the antitumor activity and safety of metformin are underway. The maximum tolerated dose and the effect of a combination of metformin and other chemotherapeutic agents need to be further investigated $(23,24)$. Since the working dose of metformin does not harm healthy cells, the drug has minimal effects on the physiological metabolism of an organism. The drug reduces the insulin level only in a hyperinsulinemic context, and has limited effect on the normal insulin level. Based on these advantages, the range of applications for metformin is expanding.

In summary, the FOXMI transcription factor is expressed in leukemia cell lines. Thiostrepton inhibits the expression of FOXM1 and $m T O R$, and may be a promising antitumor target. Metformin decreases the expression of FOXM1 in ML-2 cells. This drug accelerates apoptosis and arrests the cell cycle at the G0/G1 and G2/M phases, causing a decrease in cell proliferation. This study revealed that metformin is valuable for the treatment of leukemia, and FOXM1 may be one of its targets. Further in vivo and clinical trials need to be performed to confirm the safety of use of metformin. Use of metformin alone or combined with other chemotherapies may contribute in the treatment of leukemia.

\section{References}

1. Wang Z, Banerjee S, Kong D, Li Y and Sarkar FH: Down-regulation of Forkhead Box M1 transcription factor leads to the inhibition of invasion and angiogenesis of pancreatic cancer cells. Cancer Res 67: 8293-8300, 2007.

2. Wu QF, Liu C, Tai MH, et al: Knockdown of FoxM1 by siRNA interference decreases cell proliferation, induces cell cycle arrest and inhibits cell invasion in MHCC-97H cells in vitro. Acta Pharmacol Sin 31: 361-366, 2010. 
3. Ahmad A, Wang Z, Kong D, et al: FoxM1 down-regulation leads to inhibition of proliferation, migration and invasion of breast cancer cells through the modulation of extra-cellular matrix degrading factors. Breast Cancer Res Treat 122: 337-346, 2010.

4. Nakamura S, Hirano I, Okinaka K, et al: The FOXM1 transcriptional factor promotes the proliferation of leukemia cells through modulation of cell cycle progression in acute myeloid leukemia. Carcinogenesis 31: 2012-2021, 2010.

5. Lee MS, Hsu CC, Wahlqvist ML, Tsai HN, Chang YH and Huang YC: Type 2 diabetes increases and metformin reduces total, colorectal, liver and pancreatic cancer incidences in Taiwanese: a representative population prospective cohort study of 800,000 individuals. BMC Cancer 11: 20, 2011.

6. Green AS, Chapuis N, Lacombe C, Mayeux P, Bouscary D and Tamburini J: LKB1/AMPK/mTOR signaling pathway in hematological malignancies: from metabolism to cancer cell biology. Cell Cycle 10: 2115-2120, 2011.

7. Spender LC and Inman GJ: Phosphoinositide 3-kinase/AKT/mTORC1/2 signaling determines sensitivity of Burkitt's lymphoma cells to BH3 mimetics. Mol Cancer Res 10: 347-359, 2012

8. Feng Y, Ke C, Tang Q, et al.: Metformin promotes autophagy and apoptosis in esophageal squamous cell carcinoma by downregulating Stat3 signaling. Cell Death Dis 5: e1088, 2014.

9. Warren M, Luthra R, Yin CC, et al: Clinical impact of change of FLT3 mutation status in acute myeloid leukemia patients. Mod Pathol 25: 1405-1412, 2012.

10. Hegde NS, Sanders DA, Rodriguez R and Balasubramanian S: The transcription factor FOXM1 is a cellular target of the natural product thiostrepton. Nat Chem 3: 725-731, 2011.

11. Wonsey DR and Follettie MT: Loss of the forkhead transcription factor FoxM1 causes centrosome amplification and mitotic catastrophe. Cancer Res 65: 5181-5189, 2005.

12. Bhat UG, Halasi M and Gartel AL: Thiazole antibiotics target FoxM1 and induce apoptosis in human cancer cells. PLoS One 4: e5592, 2009.

13. Kiyoi H, Yanada M and Ozekia K: Clinical significance of FLT3 in leukemia. Int J Hematol 82: 85-92, 2005.

14. Yanada M, Matsuo K, Suzuki T, Kiyoi H and Naoe T: Prognostic significance of FLT3 internal tandem duplication and tyrosine kinase domain mutations for acute myeloid leukemia: a metaanalysis. Leukemia 19: 1345-1349, 2005.
15. Frohling S, Schlenk RF, Breitruck J, et al.: Prognostic significance of activating FLT3 mutations in younger adults (16 to 60 years) with acute myeloid leukemia and normal cytogenetics: a study of the AML Study Group Ulm. Blood 100: 4372-4380, 2002.

16. Green AS, Chapuis N, Maciel TT, et al: The LKB1/AMPK signaling pathway has tumor suppressor activity in acute myeloid leukemia through the repression of mTOR-dependent oncogenic mRNA translation. Blood 116: 4262-4273, 2010.

17. Memmott RM, Mercado JR, Maier CR, Kawabata S, Fox SD and Dennis PA: Metformin prevents tobacco carcinogen - induced lung tumorigenesis. Cancer Prev Res (Phila) 3: 1066-1076, 2010.

18. Oliveras-Ferraros C, Cufi S, Vazquez-Martin A, et al: Micro(mi) RNA expression profile of breast cancer epithelial cells treated with the anti-diabetic drug metformin: induction of the tumor suppressor miRNA let-7a and suppression of the TGF $\beta$-induced oncomiR miRNA-181a. Cell Cycle 10: 1144-1151, 2011.

19. Hirsch HA, Iliopoulos D, Tsichlis PN and Struhl K: Metformin selectively targets cancer stem cells, and acts together with chemotherapy to block tumor growth and prolong remission. Cancer Res 69: 7507-7511, 2009.

20. Wang X, Hung NJ and Costa RH: Earlier expression of the transcription factor HFH-11B diminishes induction of p21(CIP1/WAF1) levels and accelerates mouse hepatocyte entry into S-phase following carbon tetrachloride liver injury. Hepatology 33: 1404$1414,2001$.

21. Wang X, Krupczak-Hollis K, Tan Y, Dennewitz MB, Adami GR and Costa RH: Increased hepatic Forkhead Box M1B (FoxM1B) levels in old-aged mice stimulated liver regeneration through diminished p27Kip1 protein levels and increased Cdc25B expression. J Biol Chem 277: 44310-44316, 2002.

22. Sun DF, Zhang YJ, Tian XQ, Chen YX and Fang JY: Inhibition of mTOR signalling potentiates the effects of trichostatin A in human gastric cancer cell lines by promoting histone acetylation. Cell Biol Int 38: 50-63, 2014.

23. MacKenzie MJ, Ernst S, Johnson C and Winquist E: A phase I study of temsirolimus and metformin in advanced solid tumours. Invest New Drugs 30: 647-652, 2012.

24. Martin-Castillo B, Dorca J, Vazquez-Martin A, et al: Incorporating the antidiabetic drug metformin in HER2-positive breast cancer treated with neo-adjuvant chemotherapy and trastuzumab: an ongoing clinical-translational research experience at the Catalan Institute of Oncology. Ann Oncol 21: 187-189, 2010. 